\title{
Hálux valgo percutâneo: Um algoritmo de tratamento cirúrgico*
}

\section{Percutaneous Hallux Valgus: An Algorithm for the Surgical Treatment}

\author{
Igor Marijuschkin ${ }^{10}$ Matheus Levy Souza ${ }^{2 \oplus}$ José Luiz Garcia Diaz ${ }^{1}$ Paulo Carvalho $^{3}$
}

${ }^{1}$ Hospital São Lucas, Santos, SP, Brasil

${ }^{2}$ Hospital Mater Dei, Belo Horizonte, MG, Brasil

3 Hospital Ortopédico de Santana, Parede, Portugal

Rev Bras Ortop 2021;56(4):504-512.

\author{
Endereço para correspondência Igor Marijuschkin, MD, Clínica do Pé, \\ Av. Washington Luis 316, Santos, SP, 11055-001, Brasil \\ (e-mail: igormarijus@hotmail.com).
}

\begin{abstract}
Resumo
Palavras-chave

- hálux valgo

- ossos do metatarso

- procedimentos cirúrgicos minimamente invasivos

Objetivo Apresentar os resultados clínicos e radiográficos da correção cirúrgica de hálux valgo utilizando quatro técnicas percutâneas escolhidas de acordo com uma classificação radiográfica predefinida.

Métodos Avaliamos prospectivamente 112 pés em 72 pacientes com hálux valgo operado em um período de um ano. A liberação de tecido mole distal (LTMD) percutâneo e o procedimento de Akin (LTMD-Akin) foram realizados em casos leves. Em hálux valgo de leve a moderado com ângulo distal da articulação do metatarso acima de $10^{\circ}$, adicionamos a osteotomia de Reverdin-Isham (RI). Em casos moderados com incongruência articular, realizamos o chevron percutâneo (CHP). Finalmente, uma osteotomia proximal percutânea fixada (OPPF) com um parafuso, semelhante à de Ludloff, foi proposta em casos graves com ângulo intermetatarsal (AIM) acima de $17^{\circ}$. De acordo com esses critérios, foram realizados 26 LTMDs-Akin, 36 CHPs, 35 RIs e 15 OPPFs. O seguimento médio foi de 17,2 meses ( 12 a 36 meses). A média de idade em operação foi de 58,8 anos (17 a 83 anos), e $89 \%$ dos pacientes eram do sexo feminino. Resultados A média do ângulo de hálux valgo (AHV) pré-operatório e o AIM diminuíram de $21^{\circ}$ para $10,2^{\circ}$, e de $11,2^{\circ}$ para $10,3^{\circ}$, respectivamente, em casos de LTMD-Akin. Em casos de RI, a média do AHV diminuiu de $26,6^{\circ}$ para $13,7^{\circ}$, e o AIM, de $11,2^{\circ}$ para $10,3^{\circ}$; em casos de CHP, o AHV médio diminuiu de $31^{\circ}$ para $14,5^{\circ}$, o AIM diminuiu de $14,9^{\circ}$ para $10,7^{\circ}$, e a OPPF, de $39,2^{\circ}$ para $17,7^{\circ}$, e o AIM $11,8^{\circ}$ para $6,8^{\circ}$. A média do escore de tornozelo e retropé da American Orthopaedic Foot and Ankle Society (AOFAS) aumentou de 49,2 para 88,6. A taxa de complicação foi de $11 \%$. Conclusão Nosso protocolo de tratamento não difere muito dos clássicos, com resultados semelhantes. Temos como vantagem menos agressividade aos tecidos
\end{abstract}

* Trabalho desenvolvido no Hospital São Lucas, Santos, SP, Brazil.

recebido

28 de Abril de 2020

aceito

16 de Setembro de 2020
DOI https://doi.org/ 10.1055/s-0040-1721367. ISSN $0102-3616$.
(C) 2021. Sociedade Brasileira de Ortopedia e Traumatologia. All rights reserved.

This is an open access article published by Thieme under the terms of the Creative Commons Attribution-NonDerivative-NonCommercial-License, permitting copying and reproduction so long as the original work is given appropriate credit. Contents may not be used for commercial purposes, or adapted, remixed, transformed or built upon. (https://creativecommons.org/ licenses/by-nc-nd/4.0/)

Thieme Revinter Publicações Ltda., Rua do Matoso 170, Rio de Janeiro, RJ, CEP 20270-135, Brazil 


\begin{abstract}
Keywords

- hallux valgus

- metatarsal bones

- minimally invasive surgical procedures

Objective To present the clinical and radiographic results of hallux valgus surgical correction using four percutaneous techniques, chosen according to a predefined radiographic classification.

Methods We prospectively evaluated 112 feet in 72 patients with hallux valgus operated over the course of 1 year. Percutaneous distal soft tissue release (DSTR) and the Akin procedure (DSTR-Akin) were performed in mild cases. In mild to moderate hallux valgus with distal metatarsal joint angle $>10^{\circ}$, we added the Reverdin-Isham (RI) osteotomy. In moderate cases with joint incongruity, we performed the percutaneous chevron (PCH). Finally, a Ludloff-like percutaneous proximal osteotomy fixed (PPOF) with a screw was proposed in severe cases with an intermetatarsal angle (IMA) $>17^{\circ}$. According to these criteria, 26 DSTRs-Akin, 36 PCHs, 35 Rls, and 15 PPOFs were performed. The mean follow-up was of 17.2 months (range: 12 to 36 months). The mean age at operation was 58.8 years (range: 17 to 83 years), and $89 \%$ of the patients were female.

Results The mean preoperative hallux valgus angle (HVA) and the IMA decreased from $21^{\circ}$ to $10.2^{\circ}$ and from $11.2^{\circ}$ to $10.3^{\circ}$ respectively in the DSTR-Akin. In the RI, the mean HVA decreased from $26.6^{\circ}$ to $13.7^{\circ}$, and the IMA, from $11.2^{\circ}$ to $10.3^{\circ}$; in the $\mathrm{PCH}$, the mean HVA decreased from $31^{\circ}$ to $14.5^{\circ}$, and the IMA decreased $14.9^{\circ}$ to $10.7^{\circ}$; as for the PPOF, the mean HVA decreased from $39.2^{\circ}$ to $17.7^{\circ}$, and the IMA, from $11.8^{\circ}$ to $6.8^{\circ}$. The average ankle and hindfoot score of the American Orthopaedic Foot and Ankle Society (AOFAS) increased from 49.2 to 88.6. The rate of complications was of $11 \%$. Conclusion Our treatment protocol does not differ much from the classic ones, with similar results as well. We have as advantages less aggression to soft tissues and better cosmetic results. Level of Evidence: level IV, prospective case series.
\end{abstract}

moles e melhores resultados cosméticos. Nível de evidência: nível IV, série de casos prospectivos.

\section{Introdução}

A cirurgia percutânea é um conjunto de técnicas realizadas com incisões punctiformes, a fim de permitir osteotomias, exostectomias e capsulotomias, minimizando a agressão aos tecidos moles. Essa abordagem cirúrgica foi desenvolvida há 65 anos, mas só ganhou notoriedade após 1985 nos Estados Unidos, ${ }^{1}$ na década de 1990 na Europa, com Mariano Prado e Pau Golano, ${ }^{2}$ e, em 2002, na França com o Groupe de Recherche et d'Etude en Chirurgie Mini-Invasive du Pied (GRECMIP, Grupo de Pesquisa e Estudos em Cirurgia Minimamente Invasiva do Pé, em francês). ${ }^{3}$

Seguindo uma tendência mundial em diversas áreas da ortopedia para reduzir incisões e agressões cirúrgicas, a fim de diminuir complicações e comorbidades, desenvolvemos um protocolo para cirurgia de hálux valgo (HV). Utilizamos critérios radiográficos já definidos, o que não difere muito de outros autores. ${ }^{4,5}$ Foram utilizadas quatro técnicas percutâneas para a realização dos procedimentos de forma individualizada, respeitando alterações angulares, características particulares, e todos os aspectos que podem alterar a indicação cirúrgica, semelhantes aos encontrados em algoritmos relativos a procedimentos abertos.
O objetivo do presente estudo é descrever os resultados clínicos, funcionais e radiográficos do tratamento cirúrgico do HV por técnicas percutâneas.

\section{Material e Métodos}

O projeto foi apresentado e aprovado pelo Comitê de Ética em Pesquisa da instituição, com o número 0001/2020.

Avaliamos prospectivamente 112 pés em 72 pacientes com HV operados ao longo de 1 ano pelo mesmo cirurgião. As indicações cirúrgicas basearam-se na gravidade dos achados radiográficos angulares, bem como na presença de uma articulação metatarsofalangeana (MTF) congruente e aumento do ângulo articular distal do metatarso (AADM). A deformidade foi considerada leve quando o ângulo de hálux valgo (AHV) estava entre $12^{\circ}$ e $30^{\circ}$, o ângulo intermetatarsal (AIM) estava entre $8^{\circ}$ e $14^{\circ}$, e o AADM era inferior a $10^{\circ}$. O HV moderado foi considerado quando o AHV foi de até $40^{\circ}$, o AIM, entre $14^{\circ}$ e $17^{\circ}$, e o HV leve a moderado, com aumento do AADM se o AADM for superior a $10^{\circ}$, com AHV até $40^{\circ}$ e AIM até $17^{\circ}$. O HV grave foi definido como AHV acima de $40^{\circ}$ ou AIM acima de $17^{\circ}$ (-Tabela 1). 
Tabela 1 Características radiográficas e procedimento proposto

\begin{tabular}{|c|c|c|c|c|}
\hline & Hálux valgo leve & $\begin{array}{l}\text { Hálux valgo leve a } \\
\text { moderado com alto } \\
\text { ângulo articular distal } \\
\text { do metatarso }\end{array}$ & Hálux valgo moderado & Hálux valgo grave \\
\hline \multirow[t]{3}{*}{ Ângulo } & $\begin{array}{l}\text { Ângulo } \\
\text { intermetatarsal }<14^{\circ}\end{array}$ & $\begin{array}{l}\text { Ângulo } \\
\text { intermetatarsal: } 8^{\circ} \text { a } 17^{\circ}\end{array}$ & $\begin{array}{l}\text { Ângulo } \\
\text { intermetatarsal: } 12^{\circ} \text { a } 17^{\circ}\end{array}$ & Ângulo intermetatarsal $>17^{\circ}$ \\
\hline & $\begin{array}{l}\text { Ângulo de hálux } \\
\text { valgo }<30^{\circ}\end{array}$ & $\begin{array}{l}\text { Ângulo de hálux } \\
\text { valgo: } 12^{\circ} \text { a } 40^{\circ}\end{array}$ & $\begin{array}{l}\text { Ângulo de hálux } \\
\text { valgo: } 20^{\circ} \text { a } 40^{\circ}\end{array}$ & Ângulo de hálux valgo $>30^{\circ}$ \\
\hline & & $\begin{array}{l}\text { Ângulo articular distal do } \\
\text { metatarso }>10^{\circ}\end{array}$ & $\begin{array}{l}\text { Incongruência } \\
\text { da articulação }\end{array}$ & \\
\hline Técnica & $\begin{array}{l}\text { Liberação de tecido } \\
\text { mole distal e } \\
\text { procedimento de } \\
\text { Akin percutâneos }\end{array}$ & $\begin{array}{l}\text { Procedimento de } \\
\text { Reverdin-Isham }\end{array}$ & Chevron percutâneo & $\begin{array}{l}\text { Osteotomia proximal } \\
\text { percutânea }\end{array}$ \\
\hline
\end{tabular}

O AHV é o ângulo formado pelo eixo médio da diáfise da falange do hálux proximal e do eixo mecânico do primeiro metatarso. O AIM é o ângulo formado entre o eixo mecânico do primeiro e segundo metatarsos, e o AADM é o ângulo formado entre a linha perpendicular à superfície articular da cabeça do primeiro metatarso e o eixo longo do primeiro metatarso. Todas as medições angulares foram realizadas digitalmente com raios- $\mathrm{x}$ anteroposterior de carga, utilizando um software disponível comercialmente (Directview, Carestream, NY, EUA) (- Fig. 1A, B e C). Os pacientes foram avaliados utilizando o escore de tornozelo e retropé da American Orthopaedic Foot and Ankle Society (AOFAS), ${ }^{6}$ seguido de avaliações de satisfação clínica, radiográfica e de satisfação

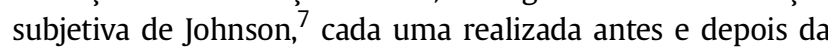
cirurgia.

A análise estatística foi feita com o programa Eviews 11 (IHS Markit, Londres, Reino Unido) para Windows, e o teste $t$ de Student emparelhado foi utilizado para avaliar os resultados com nível de significância $<0,05$.
Em casos de HV leve, realizamos exostectomia percutânea, liberação de tecido mole distal (LTMD) e osteotomia de Akin $^{8}$ (LTMD-Akin). A cirurgia foi realizada sob anestesia locorreregional ou espinhal, com o paciente em posição supina e os pés suspensos sobre a extremidade da mesa, com o pé operado apoiado no intensificador da imagem. Torniquete não era necessário.

Uma incisão foi feita na base plantar da exostose do primeiro metatarso com o bisturi Beaver 64 (BVI, Waltham, MA, EUA). Depois, removemos o periósteo com o raspador ou o bisturi, e realizamos uma exostectomia usando a broca wedge de $3.1 \mathrm{~mm}$. 0 osso foi removido da articulação por compressão manual ou curetagem, e às vezes com soro fisiológico. Em seguida, fizemos uma incisão dorsolateral na articulação metatarsofalangeal do hálux para realizar a tenotomia do tendão do adutor de hálux e capsulotomia lateral (-Fig. 2A e B). Para isso, movemos o dedo medialmente, promovendo um varo do hálux, introduzindo o bisturi Beaver com a A apontada lateralmente e
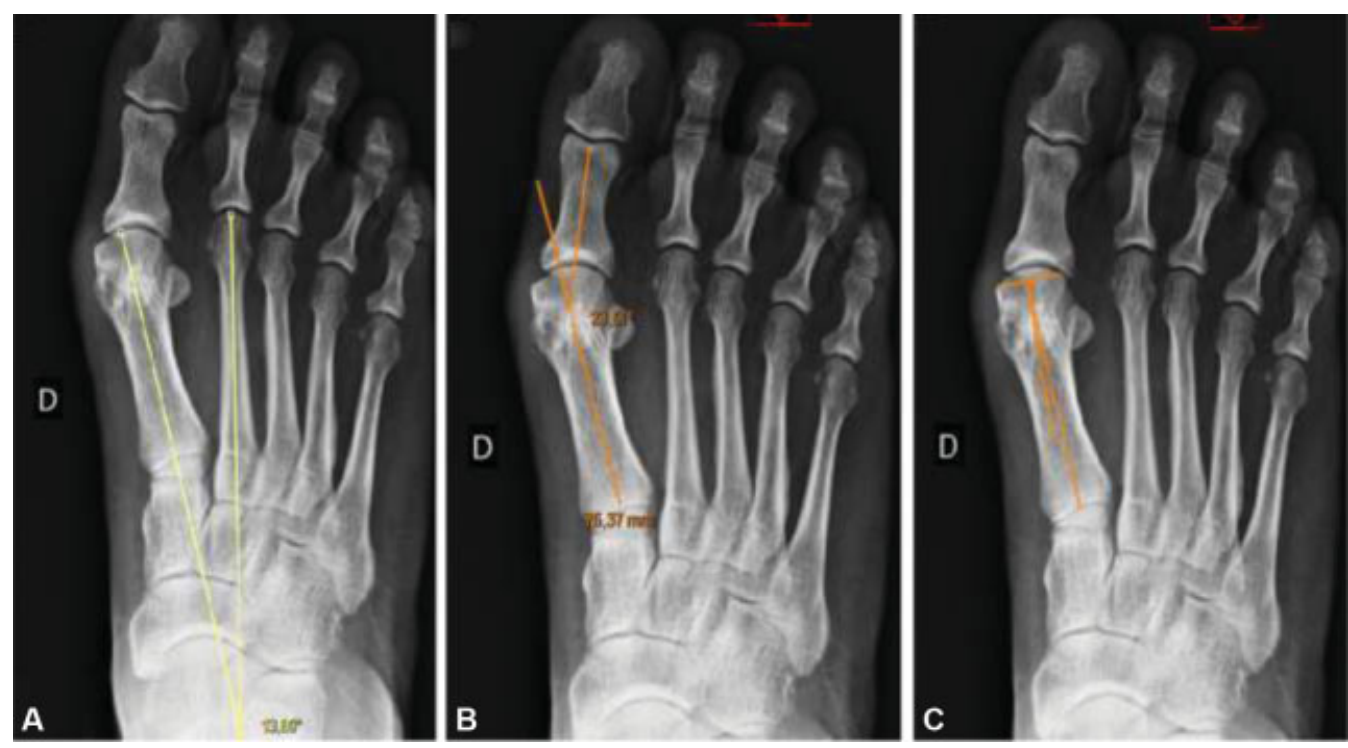

Fig. 1 Medidas angulares. (A) Ângulo de hálux valgo (AHV); (B) ângulo intermetatarsal (AIM); (C) Ângulo articular distal do metatarso (AADM). 

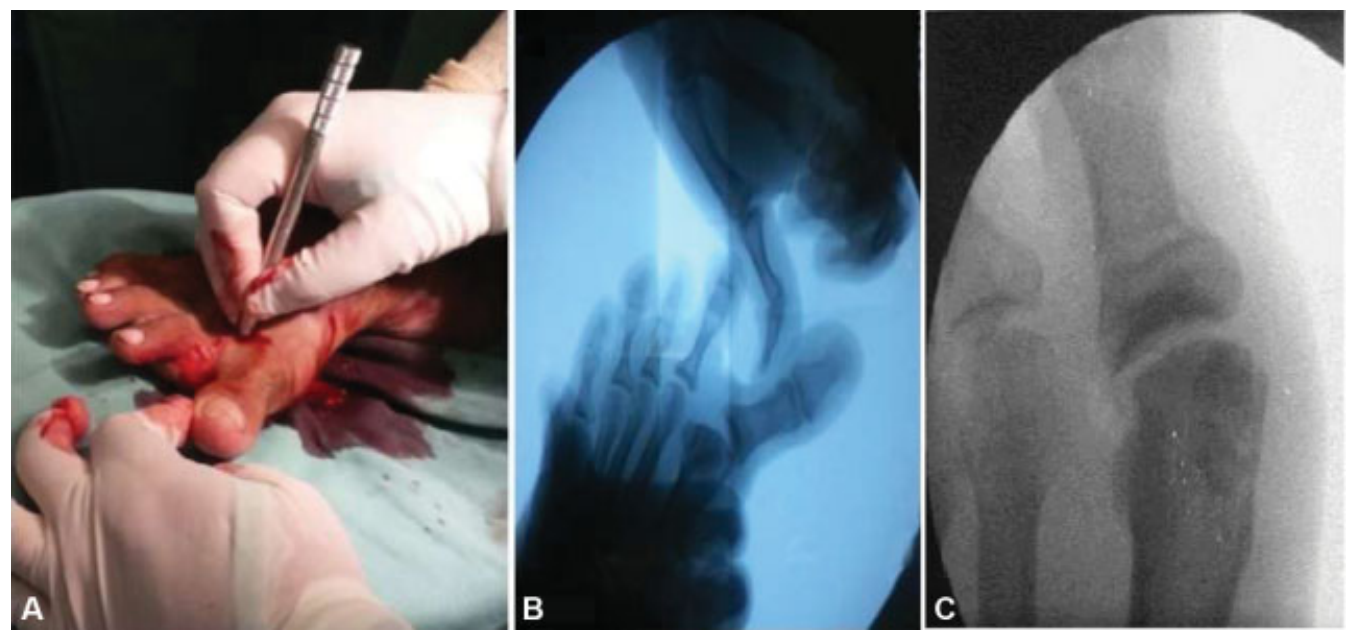

Fig. 2 (A) Incisão dorsolateral na articulação metatarsofalangeal do hálux; (B) fluoroscopia após tenotomia do tendão adutor e capsulotomia; (C) procedimento de Akin no intensificador de imagem.

profundamente, e realizamos a capsulotomia lateral e a tenotomia. Finalmente, realizamos uma incisão medial na base da falange proximal, e, com a broca wedge de $3.1 \mathrm{~mm}$, foi feita uma osteotomia incompleta para obter uma cunha de fechamento após uma compressão manual. A osteotomia foi estável, e não foi necessária fixação ( - Fig. 1C).

Em deformidades leves a moderadas com AADM alterado, executamos a técnica Reverdin-Isham ${ }^{1,3}$ (RI). Realizamos a exostectomia como descrito anteriormente, seguida de osteotomia utilizando a mesma abordagem. Introduzimos a broca de Shannon de dorsal a plantar com 45 graus de inclinação lateral na cabeça metatarsal, tomando cuidado

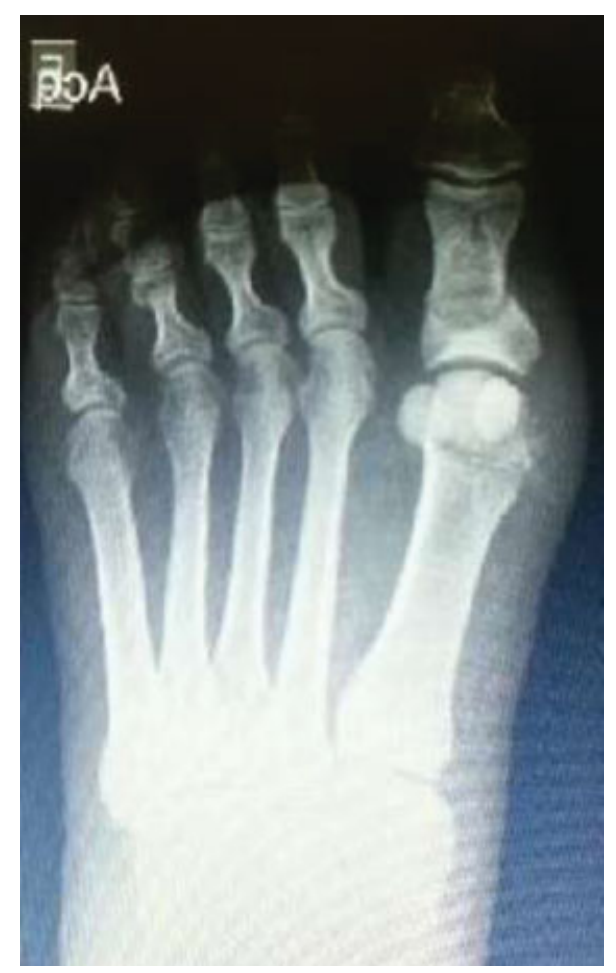

Fig. 3 Procedimento de Reverdin-Isham (RI) associada à exostectomia, tenotomia e osteotomia de Akin. para preservar o cortical lateral. Isso permite uma osteoclasia em varo, fechando a osteotomia e corrigindo o AADM, e, ao mesmo tempo, mantendo a estabilidade ( - Fig. 3). Em seguida, realizamos a liberação de tecidos moles laterais e osteotomia de Akin, como descrito anteriormente.

Em casos de HV moderado com incongruência articular, realizamos técnica de chevron percutânea (CHP) conforme descrito por Vernois. ${ }^{9}$ Foi feita uma incisão na base da exostose medial com o bisturi Beaver. Com uma broca de Shannon de $20 \mathrm{~mm}$ de comprimento, uma osteotomia em forma de $\mathrm{V}$ foi feita com o ápice ligeiramente dorsal em relação ao centro do eixo. 0 primeiro corte dorsal foi perpendicular ao chão, e o segundo, a $90^{\circ}$, paralelo ao chão. ( Fig. 4A, B e C). A broca deve ser paralela à articulação distal no plano horizontal. Com um fio de Kirschner (K) ou alavanca, a primeira cabeça metatarsal foi movida lateralmente e fixada com 1 ou 2 parafusos de $3 \mathrm{~mm}$ de diâmetro. A exostectomia foi realizada se necessário, seguida de liberação lateral de tecidos moles e procedimento de Akin.

Nos casos de HV grave, osteotomia proximal percutânea fixada (OPPF) com parafuso, semelhante à osteotomia Ludloff, ${ }^{10}$ foi realizada. 0 Beaver 64 foi usado para criar uma incisão proximal e lateral, $1 \mathrm{~cm}$ distal da articulação metatarso-cuneiforme. Destacamos o periósteo, e, com a broca de $2 \times 20 \mathrm{~mm}$ de Shannon, realizamos uma osteotomia de plantar distal oblíqua a proximal dorsal (- Fig. 5A). Com um fio K preso abaixo da cabeça metatarsal, o primeiro metatarso foi movido lateralmente e fixado com um parafuso de 3,0 mm (-Fig. 5B). Depois disso, fizemos a exostectomia percutânea, liberação lateral, e procedimento de Akin, como descrito.

O curativo é realizado com gaze e fita adesiva, mantendo o dedo em posição neutra, com $10^{\circ}$ de flexão. 0 curativo foi trocado semanalmente, durante 4 semanas, e o suporte imediato de peso com sapatos rígidos foi permitido como tolerado. Quando a patologia era bilateral, os pacientes foram operados em ambos os pés. A medição angular foi feita, e os questionários, aplicados, em seis semanas, seis meses e um ano. 

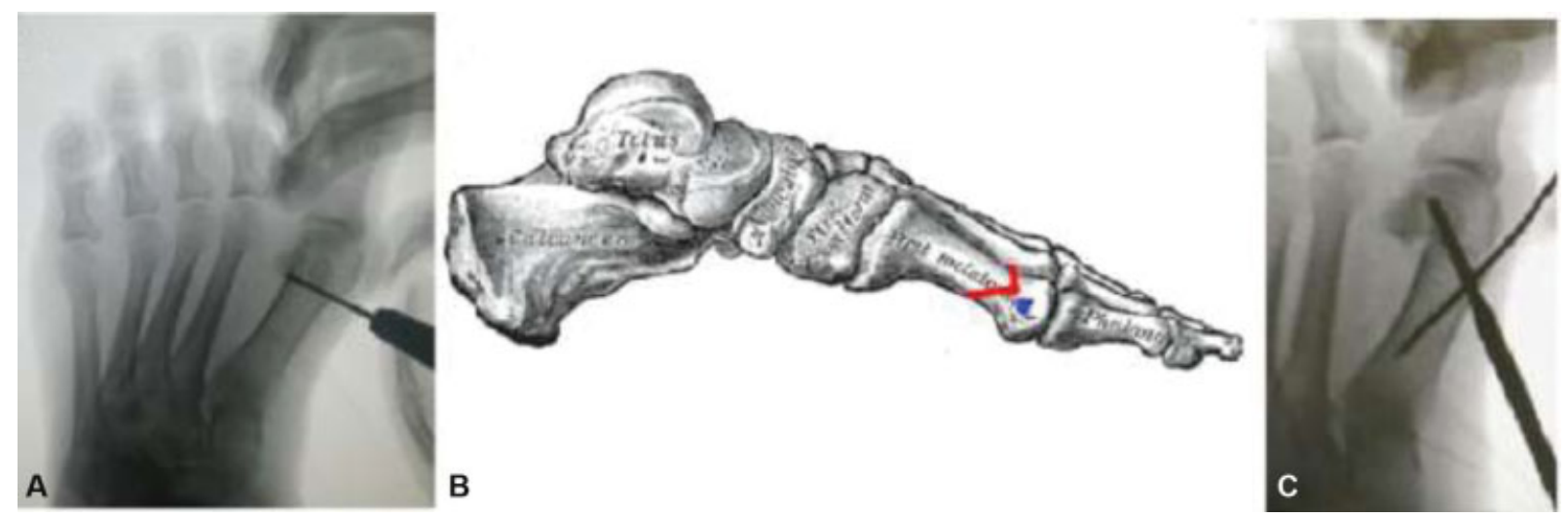

Fig. 4 (A) Controle de fluoroscopia mostrando a posição da broca, paralela à superfície articular do primeiro metatarso durante o primeiro corte para a osteotomia em chevron; (B) a linha vermelha mostra as direções para o corte em chevron; (C) fixação do chevron percutâneo com um parafuso. O fio de Kirschner é usado para ajudar na redução.
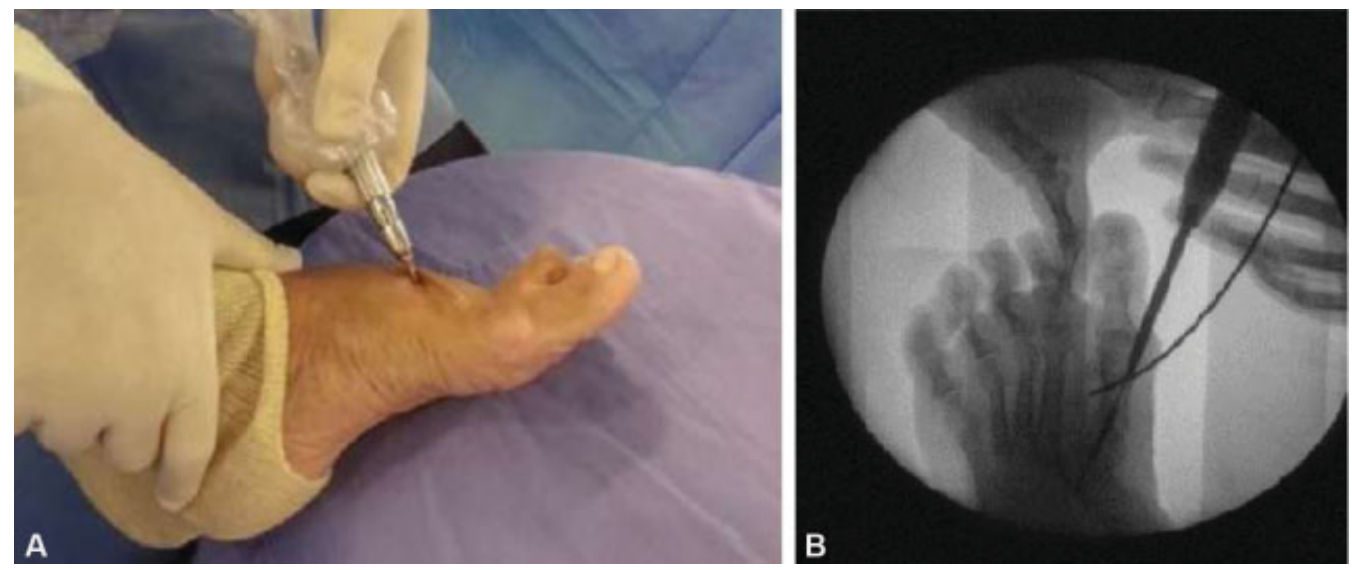

Fig. 5 (A) posição da broca para realizar a osteotomia de Ludloff; (B) fixação da osteotomia com um parafuso. O fio de Kirschner é usado para ajudar a trasladar a cabeça metatarsal.

\section{Resultados}

Foram operados 72 pacientes com idade média de 58,8 anos (gama: 17 a 83 anos), e $89 \%$ eram do sexo feminino. Eles foram divididos nas quatro categorias mencionadas anteriormente: $26 \operatorname{LTMDs}-A$ kin (23,2\%), 36 CHPs (32,1\%), 35 RIs (31,25\%), e 15 OPPFs (13,4\%). Em 54 (48\%) pés, foram necessários procedimentos adicionais para corrigir outras deformidades associadas. Realizamos osteotomias metatarsais distais minimamente invasivas em 26 pés, osteotomias corretivas de quinto joanete em 14, osteotomias corretivas de deformidades do dedo do pé em 34, neurólise em 1, e osteotomias calcâneas para pés chatos em 2 pacientes.

O seguimento médio foi de 17,2 meses (gama: 12 a 36 meses). A média do AHV pré-operatório foi de $35,1^{\circ}$ (gama: $13^{\circ}$ a $51^{\circ}$ ), e de $12,3^{\circ}$ no pós-operatório (gama: $0^{\circ}$ a $28^{\circ}$ ). Assim, obteve-se uma correção média de $22,8^{\circ}$, sendo a diferença estatisticamente significativa $(p<0,05)$. O AIM pré-operatório médio foi de $13,7^{\circ}$ (gama: $6^{\circ}$ a $23^{\circ}$ ), e de $9,3^{\circ}$ no pós-operatório (gama: $5^{\circ}$ a $\left.19^{\circ}\right)$, com correção média de $4,4^{\circ}(p<0,05)$. A média pré-operatória escore da AOFAS foi de 49,2 (gama: 34 a 60), e, no último seguimento, foi de 88,6 (gama: 45 a 100), com aumento médio de 39,4 pontos $(p<0,05)$.
Quando os grupos foram analisados separadamente, nos

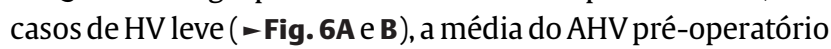
foi de $21^{\circ}$ (gama: $15^{\circ}$ a $27^{\circ}$ ), e, no último seguimento, de $10,2^{\circ}$ (gama: $4^{\circ}$ a $21^{\circ}$ ). A correção angular média foi de $10,8^{\circ}$ (gama: $7^{\circ}$ a $20^{\circ}$ ). O AIM pré-operatório médio foi de $11,2^{\circ}$ (gama: $6^{\circ}$ a $\left.15^{\circ}\right)$ e, no último seguimento, de $10,3^{\circ}\left(8^{\circ}\right.$ a $\left.13^{\circ}\right)$, com uma correção média de $0,9^{\circ}$ (gama: $0^{\circ}$ a $2^{\circ}$ ). A média do escore da AOFAS no pré-operatório foi de 55,6 (gama: 34 a 65), e, no pósoperatório, foi de 94,2 (gama: 77 a 100).

Nos casos em que foi realizada a técnica de RI ( - Fig. 7A e B), a média pré-operatória do AHV foi de $26,5^{\circ}$ (gama: $18^{\circ}$ a $36^{\circ}$ ), e, no pós-operatório, de $13,7^{\circ}$ (gama: $6^{\circ}$ a $21^{\circ}$ ), com correção média de $12,8^{\circ}$. A média do AIM foi de $13,4^{\circ}$ (gama: $11^{\circ}$ a $17^{\circ}$ ) no pré-operatório, e de $12,6^{\circ}$ (gama: $7^{\circ}$ a $17^{\circ}$ ) no pós-operatório, com correção média de $0,8^{\circ}$. A pontuação média no escore da AOFAS aumentou de 52,4 (gama: 44 a 60) para 85,3 (gama: 70 a 100) pontos. Nos casos moderados com incongruência articular em que o CHP foi indicado ( - Fig. 8A e B), o AHV diminuiu de $31^{\circ}$ (gama: $26^{\circ}$ a $40^{\circ}$ ) para $14,5^{\circ}$ (gama: $3^{\circ}$ a $26^{\circ}$ ), com uma correção média de $16,5^{\circ}$. O AIM diminuiu de $14,9^{\circ}$ (gama: $9^{\circ}$ a $17^{\circ}$ ) para $10,7^{\circ}$ (gama: $8^{\circ}$ a $16^{\circ}$ ), com uma correção média de $4,2^{\circ}$. A pontuação média no escore da AOFAS aumentou de 42 para 87,1 pontos. 


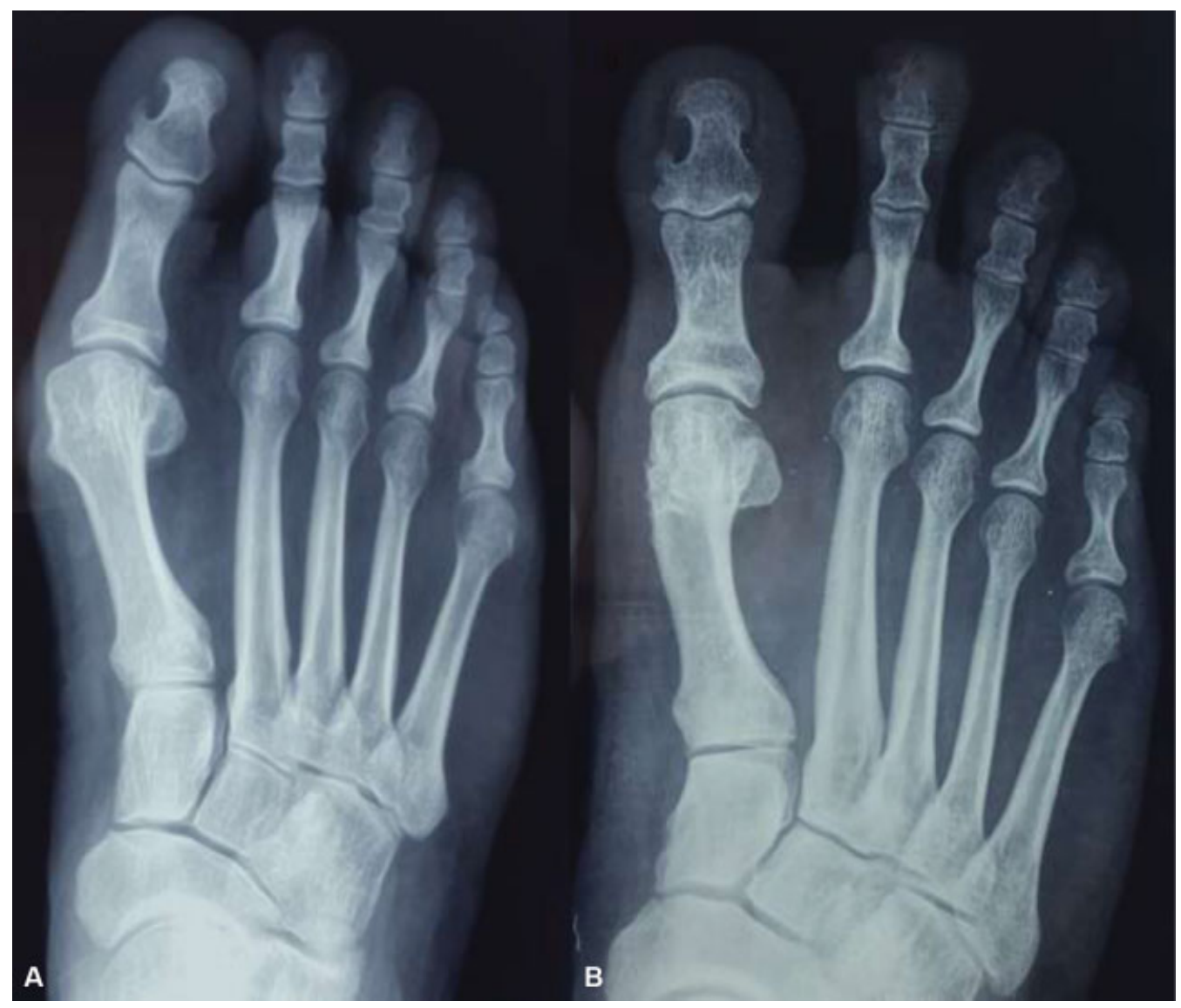

Fig. 6 (A) caso leve no pré-operatório. (B) caso leve no pós-operatório (exostectomia + liberação de tecido mole distal + procedimento de Akin).

Em casos graves, em que a OPPF foi executada ( - Fig. 9A e B), a média pré-operatória do AHV diminuiu de $39,2^{\circ}$ (gama: $30^{\circ}$ a $51^{\circ}$ ) para $17,7^{\circ}$ (gama: $8^{\circ}$ a $28^{\circ}$ ), com correção média de $21,5^{\circ}$. A média do AIM no pré-operatório diminuiu de $18,4^{\circ}$ (gama: $15^{\circ}$ a $23^{\circ}$ ) para $11,8^{\circ}$ (gama: $7^{\circ}$ a $17^{\circ}$ ), com uma correção média de $6,8^{\circ}$. A média do escore da AOFAS no pré-operatório foi de 41,8 (gama: 34 A 50) e aumentou para 88 (gama: 70 a 100) pontos no último seguimento. Um paciente ficou insatisfeito com o desfecho devido a recidiva.

Em relação às complicações, houve 6 casos de remoção do parafuso (3 CHPs e 3 OPPFs), uma fístula (CHP), 3 casos de rigidez articular (2 RIs e $1 \mathrm{CHP}$ ), 2 casos de edema residual após 1 ano de cirurgia (OPPF), 2 casos de metatarsalgia de transferência (1 CHPe 1 OPPF), 2 não uniões de DMMOs assintomáticas após 1 ano, e 1 broca quebrada que não pôde ser removida. Dois pacientes apresentaram calo sintomático, causado pela luxação dorsal da primeira cabeça metatarsal (1 RI e 1 OPPF), 1 recorrência em menos de 1 ano (OPPF), e 1 neuropatia não transitória (LTMD-Akin). Estes representam $18 \%$ do total de casos. 0 percentual de complicações foi proporcionalmente maior nos casos graves (33\%; OPPF), seguido pelos moderados (18\%; CHP), intermediários (17,6\%; RI) e leves (12\%; LTMD-Akin).

\section{Discussão}

Várias técnicas para o tratamento cirúrgico de HV foram descritas. Não acreditamos que haja uma técnica que possa resolver todos os casos. O presente estudo é baseado em conceitos modernos de tratamento, que originaram protocolos baseados em mudanças clínicas e radiográficas. ${ }^{11,12}$ Nosso protocolo baseia-se nos critérios e técnicas já estabelecidos para procedimentos abertos. A principal diferença é a aplicação deste protocolo com técnica percutânea e fixação estável, preservando a biologia local. ${ }^{13}$

Em casos leves, optamos por realizar LTMD-Akin, segundo Kayali et al., ${ }^{14}$ pois a liberação de tecidos moles reequilibra as forças deformantes e, com ela, pode-se obter boas correções em casos de HV leve. Este procedimento é feito somente quando o AIM é inferior a $15^{\circ}$. No presente estudo, não houve recidiva com até 28,6 meses de seguimento. Colloff e Weitz ${ }^{15}$ e Martinez-Nova et al. $^{16}$ também realizaram LTMD-Akin percutânea no tratamento de HV leve a moderado, com resultados semelhantes no curto prazo. Pansini et al. ${ }^{17}$ utilizaram osteotomia de Akin em 89,1\% das cirurgias de HV, destacando a importância deste procedimento para a correção da deformidade em combinação com outras técnicas.

Obtivemos resultados semelhantes em comparação com os já publicados na literatura, na qual a técnica de Austin é a mais popular. ${ }^{18,19}$ Basile et al. ${ }^{20}$ compararam o procedimento de Akin associado com chevron com LTMD-Akin, e descreveram resultados semelhantes, com leve vantagem radiográfica para chevron com Akin.

Em casos leves a moderados, que requerem correção de AADM, consideramos o chevron ${ }^{21}$ biplano a técnica aberta mais adequada. Realizamos a técnica de $\mathrm{RI}^{4}$ com bons resultados como já descrito na literatura em um estudo multicêntrico. ${ }^{5}$ 
510 Hálux valgo percutâneo: Um algoritmo de tratamento cirúrgico Marijuschkin et al.

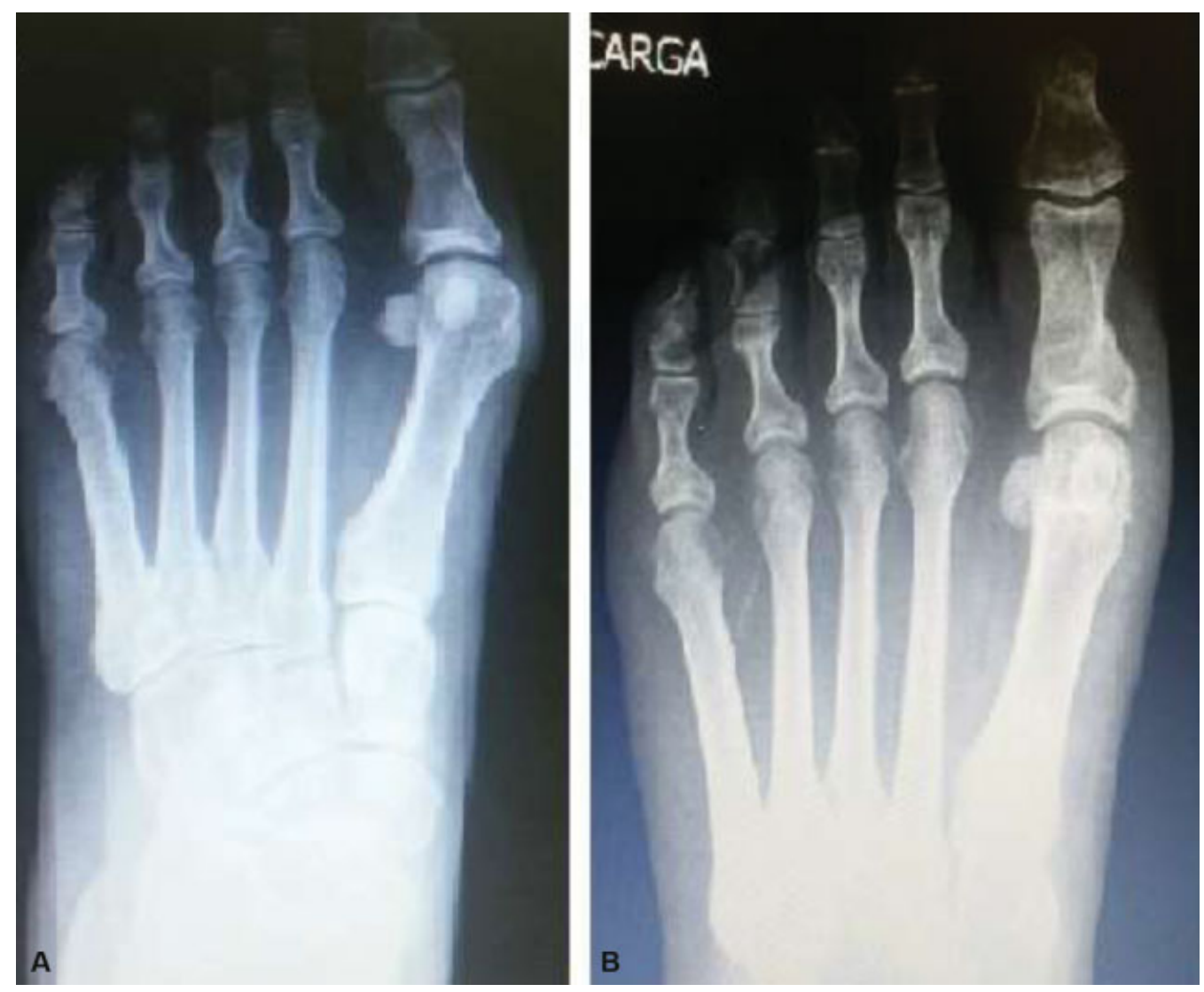

Fig. 7 (A) caso moderado no pré-operatório com AADM qalterado; (B) procedimento de RI pós-operatória com exostectomia + tenotomia + procedimento de Akin.
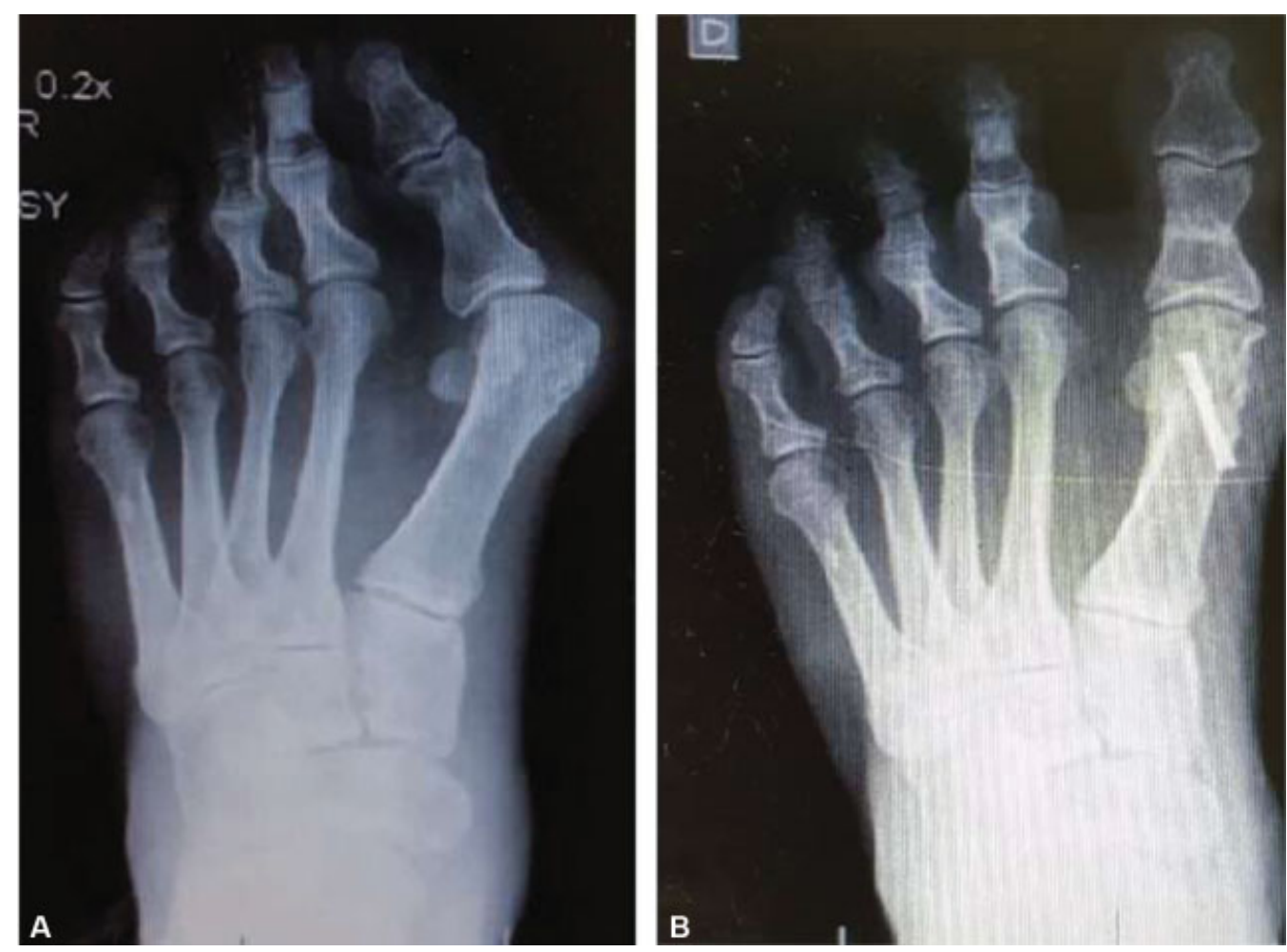

Fig. 8 (A) Pré-operatório de caso moderado com incongruência articular; (B) pós-operatório depois de chevron percutâneo. 


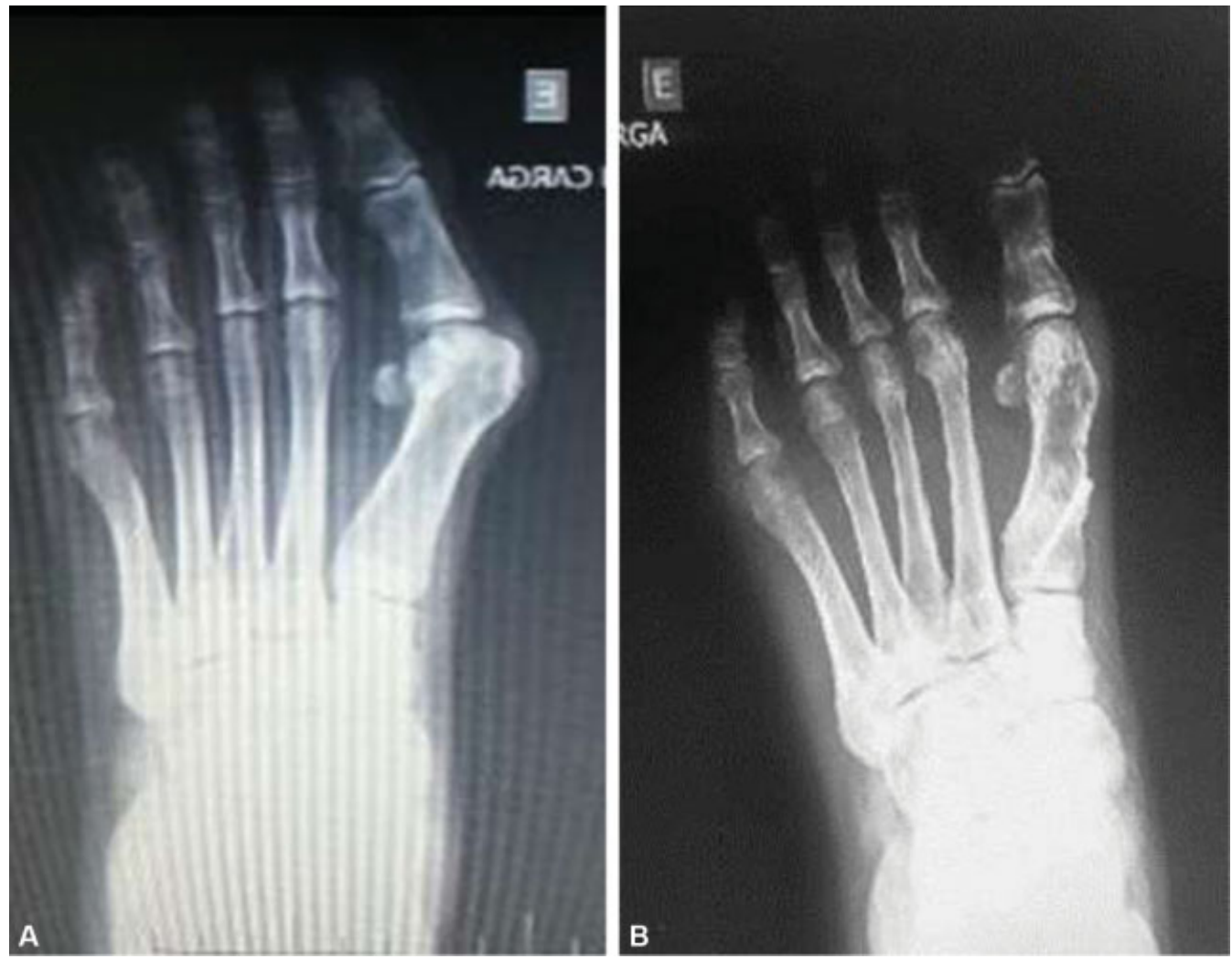

Fig. 9 (A) Pré-operatório de caso grave de hálux valgo; (B) pós-operatório de osteotomia similar ao procedimento de Ludloff.

Tabela 2 Valores pré- e pós-operatórios do AHV e do AIM com correção média e do escore da AOFAS

\begin{tabular}{|l|l|l|l|l|l|l|l|l|}
\hline & Resultados & & & & & & & \\
\hline Procedimento & AHV inicial & AHV final & AHV (CM) & AIM inicial & AIM final & AIM (CM) & AOFAS Inicial & AOFAS Final \\
\hline LTMD-Akin & $21^{\circ}$ & $10,2^{\circ}$ & $10,8^{\circ}$ & $11,2^{\circ}$ & $10,3^{\circ}$ & $0,9^{\circ}$ & 55,6 & 94,2 \\
\hline Reverdin-Isham & $26,5^{\circ}$ & $13,7^{\circ}$ & $12,8^{\circ}$ & $13,4^{\circ}$ & $12,6^{\circ}$ & $0,8^{\circ}$ & 52,4 & 85,3 \\
\hline $\begin{array}{l}\text { Chevron } \\
\text { percutâneo }\end{array}$ & $31^{\circ}$ & $14,5^{\circ}$ & $16,5^{\circ}$ & $14,9^{\circ}$ & $10,7^{\circ}$ & $4,2^{\circ}$ & 42 & 87,1 \\
\hline OPPF & $39,2^{\circ}$ & $17,7^{\circ}$ & $21,5^{\circ}$ & $18,4^{\circ}$ & $11,8^{\circ}$ & $6,8^{\circ}$ & 41,8 & 88 \\
\hline Total & $35,1^{\circ}$ & $17,2^{\circ}$ & $22,8^{\circ}$ & $13,7^{\circ}$ & $9,3^{\circ}$ & $4,4^{\circ}$ & 49,2 & 88,6 \\
\hline
\end{tabular}

Abreviaturas: AHV, ângulo de hálux valgo; AIM, ângulo intermetatarsal; AOFAS, escore de tornozelo e retropé da American Orthopaedic Foot and Ankle Society; CM, correção média; LTMD-Akin, liberação de tecido mole distal percutâneo e procedimento de Akin; OPPF, osteotomia proximal percutânea fixada com parafuso.

Em casos de HV moderado a grave com incongruência articular, escolhemos o CHP como descrito por Vernois e Redfern. ${ }^{22}$ Marijuschkin et al. ${ }^{23}$ demonstraram a vantagem dessa técnica com baixa taxa de complicações e alta taxa de satisfação do paciente em comparação com as técnicas convencionais. Lam et al., ${ }^{24}$ em estudo comparativo com a osteotomia em cachecol, destacaram a vantagem no tempo cirúrgico e na dor pós-operatória. Brogan et al. ${ }^{25}$ compararam o chevron convencional e o CHP, e afirmaram que não houve diferenças nas complicações e resultados, sugerindo vantagem de curto prazo para o CHP.

Em casos graves, optou-se pela OPPF com parafusos, semelhante à osteotomia Ludloff, permitindo a correção do AIM. Segundo Saxena e McCammon, ${ }^{26}$ que realizaram a osteotomia de Ludloff convencional, essa técnica tem a vantagem de proporcionar melhor estabilidade do que o chevron proximal e a osteotomia crescente. Lavigne et al. ${ }^{27}$ descreveram uma osteotomia percutânea tripla para a correção de deformidades graves, fazendo três cunhas de encurtamento fixo, o que não foi nossa escolha, devido à grande dificuldade técnica. Vernois e Redfern ${ }^{13}$ realizaram osteotomia de CHP e osteotomias de base metatarsal incompletas para o tratamento de HV grave, ambas com bons resultados; estas também podem ser boas opções. Quanto às técnicas abertas, Mann et al. ${ }^{28}$ realizaram osteotomia proximal e liberação lateral com resultados semelhantes e com elevação de $28 \%$ da cabeça metatarsal, complicações elevadas típicas de casos graves. Considerando a osteotomia de Ludloff, Myerson ${ }^{29}$ descreveu complicações em 22 de 75 
pés, e considerou esses bons resultados, com boas correções radiográficas e boa satisfação do paciente.

A cirurgia percutânea tem muitas vantagens, especialmente considerando suas características minimamente invasivas. Conforme apresentado por Lara et al., ${ }^{30}$ é menos dolorosa do que a cirurgia aberta, e tem um resultado cosmético melhor, e resultados gerais muito semelhantes. Estes podem ser confirmados em nossos resultados pela elevada satisfação do paciente e pelos altos escores da AOFAS.

O presente estudo tem algumas limitações em relação ao formato de série de casos. Por um lado, não tivemos um grupo de controle de cirurgia aberta, e o acompanhamento foi limitado. Por outro lado, trata-se de um trabalho prospectivo com um número significativo de pacientes operados em um período de um ano pelo mesmo cirurgião. Acreditamos que novas pesquisas são necessárias para estabelecer esse protocolo, mas nosso estudo é inicial, e mostra que a técnica percutânea, com todos os seus benefícios, poderia ser aplicada em substituição aos procedimentos abertos, com resultados muito semelhantes. Em estudos futuros, sugerimos um acompanhamento mais longo para avaliar a recorrência potencial e uma amostra mais numerosa, para confirmar o protocolo.

\section{Conclusão}

As técnicas cirúrgicas descritas foram eficazes no tratamento do HV, com melhora no escore da AOFAS, alta satisfação do paciente, e boa correção radiográfica.

Com a cirurgia percutânea e a cirurgia convencional, pode-se tratar o $\mathrm{HV}$ em relação às características peculiares de cada caso, considerando o conhecimento radiográfico e clínico já adquirido com os estudos clássicos. A cirurgia percutânea tem como vantagem menos agressividade aos tecidos moles, proporcionando melhores resultados cosméticos e recuperação menos dolorosa.

\section{Suporte Financeiro}

Não houve suporte financeiro de fontes públicas, comerciais, ou sem fins lucrativos.

\section{Conflito de Interesses}

Os autores declaram não haver conflito de interesses.

\section{Referências}

1 Isham SA. The Reverdin-Isham procedure for the correction of hallux abducto valgus. A distal metatarsal osteotomy procedure. Clin Podiatr Med Surg 1991;8(01):81-94

2 De Prado M, Ripoll PL, Golano P. Hallux valgus. In: Percutaneous foot surgery. Barcelona: Masson; 2003:57-94

3 Bauer T, Biau D, Lortat-Jacob A, Hardy P. Percutaneous hallux valgus correction using the Reverdin-Isham osteotomy. Orthop Traumatol Surg Res 2010;96(04):407-416

4 Coughlin MJ. Hallux valgus. J Bone Joint Surg Am 1996;78(06):932-966

5 Coughlin MJ, Saltzman CL, Anderson RB. Mann's Surgery of the Foot and Ankle. 9th ed. Philadelphia: Elsevier/Saunders; 2014

6 Rodrigues RC, Masiero C, Mizusaki JM, et al. Translation, cultural adaptation and validation of American Orthopedic Foot and Ankle
Society (AOFAS) Ankle-Hindfoot scale. Acta Ortop Bras 2008;16 (02):107-111

7 Johnson KA, Cofield RH, Morrey BF. Chevron osteotomy for hallux valgus. Clin Orthop Relat Res 1979;(142):44-47

8 Akin 0 . The treatment of hallux valgus - a new operative procedure and its results. Med Sentinel 1925;33:678-683

9 Vernois J. L'ostéotomie et chevron percutané du premier métatarsien. In: Cazeau C, ed. Chirurgie mini-invasive et percutanée du pied. Paris: Sauramps Médical; 2009:97-104

10 Ludloff K. Die beseitgung des hallux valgus Dirch die schrage plantodorsale osteotomei des metatarsalus I. Arch Klin Chir 1918; 110:364-387

11 Robinson AH, Limbers JP. Modern concepts in the treatment of hallux valgus. J Bone Joint Surg Br 2005;87(08):1038-1045

12 Trnka HJ. Osteotomies for hallux valgus correction. Foot Ankle Clin 2005;10(01):15-33

13 Vernois J, Redfern DJ. Percutaneous chevron: The union of classic stable fixed approach and percutaneous technique. Fuss \& Sprunggelenk 2013;11(02):70-75

14 Kayali C, Ozturk H, Agus H, Altay T, Hancerli O. The effectiveness of distal soft tissue procedures in hallux valgus. J Orthop Traumatol 2008;9(03):117-121

15 Colloff B, Weitz EM. Proximal phalangeal osteotomy in hallux valgus. Clin Orthop Relat Res 1967;54(54):105-113

16 Martínez-Nova A, Sánchez-Rodríguez R, Leal-Muro A, SánchezBarrado E, Pedrera-Zamorano JD. Percutaneous distal soft tissue release-akin procedure, clinical and podobarometric assessment with the BioFoot in-shoe system: a preliminary report. Foot Ankle Spec 2008;1(04):222-230

17 Pansini JV, Matunaga RY, Aguiar A, Buchen EC. Clinical and radiographic analysis of hallux valgus treatment by distal Chevron osteotomy. Rev ABTPe 2008;2(01):17-22

18 Coull R, Stephens M. Operative decision making in hallux valgus. Curr Orthop 2002;16(03):180-186

19 Trnka HJ, Zembsch A, Wiesauer H, Hungerford M, Salzer M, Ritschl P. Modified Austin procedure for correction of hallux valgus. Foot Ankle Int 1997;18(03):119-127

20 Basile A, Battaglia A, Campi A. Comparison of chevron-Akin osteotomy and distal soft tissue reconstruction-Akin osteotomy for correction of mild hallux valgus. Foot Ankle Surg 2000;6(03):155-163

21 Nery C, Barroco R, Réssio C. Biplanar chevron osteotomy. Foot Ankle Int 2002;23(09):792-798

22 Vernois J, Redfern DJ. Percutaneous Surgery for Severe Hallux Valgus. Foot Ankle Clin 2016;21(03):479-493

23 Marijuschkin I, Camargo AA, Diaz JL, Magalhães SL. Surgical treatment of mild and moderate hallux valgus by percutaneous Chevron technique. Tobillo Pie 2015;7(01):21-28

24 Lam P, Lee M, Xing J, Di Nallo M. Percutaneous Surgery for Mild to Moderate Hallux Valgus. Foot Ankle Clin 2016;21(03):459-477

25 Brogan K, Lindisfarne E, Akehurst H, Farook U, Shrier W, Palmer S. Minimally Invasive and Open Distal Chevron Osteotomy for Mild to Moderate Hallux Valgus. Foot Ankle Int 2016;37(11):1197-1204

26 Saxena A, McCammon D. The Ludloff osteotomy: a critical analysis. J Foot Ankle Surg 1997;36(02):100-105

27 Lavigne C, Rasmont Q, Hoang B. Percutaneous double metatarsal osteotomy for hallux valgus correction of severe deformity. Acta Orthop Belg 2011;77(04):516-521

28 Mann RA, Rudicel S, Graves SC. Repair of hallux valgus with a distal soft-tissue procedure and proximal metatarsal osteotomy. A longterm follow-up. J Bone Joint Surg Am 1992;74(01):124-129

29 Myerson MS. The Ludloff osteotomy. In: Joint Meeting of the American Orthopaedic Foot \& Ankle Society and the Japanese Society for Surgery of the Foot. Hawaii, November 13-15, 1997

30 Lara LC, Montesi Neto DJ, Guerra RR, Marques RF, Bicudo LR. Postoperative pain behavior of the hallux valgus using conventional, minimally invasive and percutaneous techniques. Rev ABTPe 2011;5(02):81-86 